\title{
A Synthesis of emerging data collection technologies and their impact on traffic management applications
}

\author{
Constantinos Antoniou • Ramachandran Balakrishna • \\ Haris N. Koutsopoulos
}

Received: 26 December 2010 / Accepted: 8 September 2011 /Published online: 4 October 2011

(C) The Author(s) 2011. This article is published with open access at SpringerLink.com

\begin{abstract}
Purpose The objective of this research is to provide an overview of emerging data collection technologies and their impact on traffic management applications.

Methods Several existing and emerging surveillance technologies are being used for traffic data collection. Each of these technologies has different technical characteristics and operating principles, which determine the types of data collected, accuracy of the measurements, levels of maturity, feasibility and cost, and network coverage. This paper reviews the different sources of traffic surveillance data currently employed, and the types of traffic management applications they may support.

Results Automated Vehicle Identification data have several applications in traffic management and many more are certain to emerge as these data become more widely available, reliable, and accessible. Representative examples in this field are presented. Furthermore, the fusion of condition information with traffic data can result in better and more responsive dynamic traffic management applications with a richer data background.
\end{abstract}

C. Antoniou ( $\square)$

Laboratory of Transportation Engineering,

National Technical University of Athens,

Zografou, Greece

e-mail: antoniou@central.ntua.gr

R. Balakrishna

Caliper Corporation,

Newton, MA, USA

e-mail: rama@caliper.com

H. N. Koutsopoulos

Division of Transport and Logistics,

The Royal Institute of Technology,

Stockholm, Sweden

e-mail: hnk@infra.kth.se
Conclusions The current state-of-the-art of traffic modeling is discussed, in the context of using emerging data sources for better planning, operations and dynamic management of road networks.

Keywords Emerging data sources · Traffic surveillance · Traffic management $\cdot$ Data fusion

\section{Introduction}

Growing traffic levels and their related externalities have prompted research into methods to alleviate urban traffic congestion. Intelligent transportation systems (ITS) are being widely deployed to better manage and operate existing transportation infrastructure. The broad objectives of advanced traffic management systems (ATMS), advanced traveler information systems (ATIS) and advanced public transportation systems (APTS) are the use of sophisticated technologies to improve the efficiency of current transportation modes, maximize capacity, minimize delays and improve system reliability. Several software programs have been developed to support these initiatives. Such systems simulate road network performance at various levels of detail, optimize signal cycles, estimate and predict real-time conditions and generate consistent, anticipatory route guidance [19, $20,42,62]$.

The development of advanced traffic optimization software has been motivated by the large-scale deployment of traffic surveillance technologies. Towns and cities are increasingly installing sophisticated sensor networks to automatically and routinely collect and archive timevarying traffic data. Such sensors vary widely in their operating principles, resulting in a diverse array of potential data. Each type of data possesses strengths that may be 
exploited by certain types of traffic management applications. In this paper, we review current and emerging data collection technologies, and classify them according to (a) the type(s) of data they collect and (b) their primary applications. This discussion is intended as a synthesis of the current state-ofthe-practice of traffic data collection, as well as the state-ofthe-art of traffic modeling and systems management. Therefore, the emphasis is not on the technologies themselves, but specifically on the use of the collected data for traffic management purposes.

The remainder of the paper is organized as follows. The following section provides a classification of traffic sensor technologies based on their operating principles and the traffic metrics they can potentially collect. The subsequent section outlines the emerging dynamic traffic assignment models, followed by a section highlighting the aspects of these models that can benefit from richer data. The next section details a number of opportunities provided in light of the new traffic data collection technologies, while the concluding section includes a summary of the current state-of-the-art.

\section{Traffic sensor technologies}

Based on their functionality traffic sensors can be categorized as point, point-to-point and area-wide.

\subsection{Point sensors}

Point sensors are the most widely used type of detector in use today. (Inductive) loop detectors have been in use for decades, while other technologies, including radar, infrared, acoustic, and video sensors have also been developed. This category includes:

- Inductive loop detectors: By far the most widely deployed sensor technology, loop detectors are typically low cost sensors but installation and maintenance are disruptive to traffic, and there are potentially serious reliability/accuracy issues. Due to their ubiquity, researchers have developed ways to use them for vehicle classification [50] and vehicle re-identification $[28,48,51]$. Recently, devices that can perform similar functions with higher accuracy and reliability, easier installation, lower maintenance and longer life span have been introduced (e.g. sensysnetworks.com).

- Radar/Infrared/Microwave/Acoustic/Ultrasonic sensors: These non-intrusive roadside technologies cause minimal disruption to normal traffic operations and do not need to be installed in or on the pavement. They are generally mounted overhead or to the side of the pavement, often on pre-existing structures. Most of these sensors are cost competitive with inductive loop detectors, if not cheaper [49].

- Video image detection systems: Closed-circuit television (CCTV) cameras record traffic images, which are in turn analyzed by machine vision software to monitor freeway conditions, collect data at intersections for traffic control purposes, detect incidents and classify vehicles. These systems may have high initial costs, but require little maintenance (which minimizes traffic disruptions). They also do not suffer from the reliability issues associated with loop detectors [53]. However, the performance of video image detection systems are affected by adverse weather conditions (such as rain, fog) and limited visibility (e.g. night) [46].

- Weigh-in-motion (WIM) systems: WIM systems allow for the unobtrusive and continuous collection of vehicle weight information [64].

\subsection{Point-to-point sensors}

These emerging technologies detect vehicles at multiple locations as they traverse the network. This supports reidentification and tracking, which may (under certain conditions) provide point-to-point travel times, route choice fractions, paths, and OD flows. Examples of technologies in this category include:

- Automated Vehicle Identification (AVI) systems: The underlying principle is based on the identification of individual (probe) vehicles equipped with an appropriate device, at various locations in the network $[2,3]$. Hellinga and $\mathrm{Fu}$ [36] provide an assessment of the expected accuracy of probe vehicle travel time reports, including the various sources of bias that can affect AVI-based travel time collection.

- Vehicle identification without driver "cooperation": One disadvantage of the AVI approach is that it requires that the vehicles be equipped with special electronic tags (e.g. those used for electronic tolling). Recently, approaches that leverage the Bluetooth and Wi-Fi radios on cell-phones carried by drivers in passing vehicles have been developed [16, 35, 55]. The advantage of these approaches is that they merely "ping" the Bluetooth or Wi-Fi adapters for their MAC hardware address, thus only relying that a phone with an activated Bluetooth or Wi-Fi adapter is within range. Since the vehicle-to-device correspondence is not necessarily one-to-one (drivers may not carry a Bluetooth-equipped device, or a vehicle may contain more than one Bluetooth device), such data collection methods are more appropriate for measuring quantities such as speeds and route choice fractions, but not counts. 
- License plate recognition: CCTV cameras record license plates, which are recognized through special Optical Character Recognition (OCR) software, and transferred to a central system that matches subsequent detections of particular vehicles [31]. These technologies are also limited by the susceptibility of cameras to weather and lighting conditions [46].

\subsection{Area-wide sensors}

While the examples listed above are sensors mainly dedicated to traffic applications a number of opportunistic sensors are receiving attention for their potential to provide traffic data for real time traffic monitoring, traffic information, and traffic prediction. Cell phones, smart-phones, and Global Positioning Systems (GPS) are typical examples of such sensors. Furthermore, this category includes promising technologies that are still under research and include airborne sensors such as unmanned aircraft continuously flying over and surveying a traffic network. The traffic information (collected e.g. using photogrammetry, videorecording, and Light Detection and Ranging (LIDAR) techniques), is communicated to the TMC via wireless communication networks [57].

GPS devices in the vehicle collect location information, which is transmitted to a central facility for processing. A downside to this technology is that a wireless telecommunication connection (e.g. through a cell phone) is required for the transfer of information from the vehicle to the TMC [56].

Wireless service providers can automatically collect geo-location data of wireless phones, which can be used to extract flow and speed information. The concept is still at an experimental stage, but applications are emerging, e.g. for the measurement of traffic speeds and travel times [15] and the identification of spatial and temporal congestion characteristics [18]. The accuracy of the technology is limited by several parameters (including the size and density of the GSM cells) [38].

Technologies such as Automated Vehicle Location (AVL) are in use in the transit industry. Modern AVL systems are based on GPS or differential GPS, often augmented by dead reckoning, for collection of data on vehicle location, speed and other information [32, 33, 52].

The Mobile Century project aimed at a proof of concept for using smartphones with GPS capabilities to collect traffic data and use them for real time traffic estimation and prediction. The field test involved 100 cars. It is to be followed by Mobile Millennium, a large-scale freeway experiment with the same objectives. The study will focus on commuters in the San Francisco Bay Area [60].

\subsection{Summary}

Table 1 summarizes the data collection capabilities of the main sensor technologies described above. Any given traffic modeling application is likely to be supported by a wide range of traffic data types. The accuracy of the model may in fact be significantly enhanced by using more than one type of data. It has been shown, for example, that the calibration of DTA models benefits from the addition of speed or density data to the link counts that are typically used in practice (cf. Section 3). There may be specific instances, however, when a particular data type can prove invaluable. One such instance (from Table 1) is vehicle classification data that can support the development of traffic model inputs by vehicle type (cars, vans, trucks, buses, etc.), thereby increasing the fidelity of the model and its applications.

Emerging data collection technologies provide a multitude of rich data from diverse data sources. This data creates new opportunities in dynamic traffic management, as well as other aspects of traffic simulation and prediction. The relationship between data availability and applications is bi-directional. Some technologies have been conceived with specific applications in mind (e.g. license plate recognition and transponder-based AVI), while others have evolved as improvements of existing technologies. In any case, their emergence has led to applications that were not possible before, e.g. dynamic congestion pricing in urban areas.

The additional data that are collected are useful not only on their own merit, but also as used in applications to improve the accuracy of the estimation and prediction of OD flows. In this paper, several applications that can benefit from such data are considered, and indicative results are presented. The experiences are not only useful in demonstrating the potential benefits of such emerging data collection techniques, but can also be used to fine-tune and improve their deployments. Network configuration as well as the configuration of the sensor infrastructure can considerably affect the performance of the data collection effort and therefore the effectiveness of the supported applications. For example, the configuration of the ETC sensors forming an automatic vehicle identification network may have considerable impact on system performance. Chen et al. [26] formulate the problem of optimal location of AVI sensors as a multi-objective integer-optimization problem with the following objectives: minimize the number of AVI readers, maximize the OD coverage and maximize the number of trips covered by the system. The authors apply a distance-based genetic algorithm to solve the problem by explicitly generating the non-dominated solutions. Mirchandani and $\mathrm{He}$ [47] formulate a 0-1 mathematical program to determine the routes on which to 
Table 1 Main types of data collected by each sensor type

\begin{tabular}{|c|c|c|c|c|c|c|c|c|c|}
\hline & & \multicolumn{8}{|c|}{ Data collection technologies } \\
\hline & & \multicolumn{3}{|c|}{ Point sensors } & \multicolumn{3}{|c|}{ Point-to-point } & \multicolumn{2}{|c|}{ Area-wide } \\
\hline & & $\begin{array}{l}\text { Loop } \\
\text { detectors }\end{array}$ & $\begin{array}{l}\text { Radar/infrared/acoustic } \\
\text { sensors }\end{array}$ & $\begin{array}{l}\text { CCTV } \\
\text { cameras }\end{array}$ & $\begin{array}{l}\text { License } \\
\text { plate } \\
\text { recognition }\end{array}$ & $\begin{array}{l}\text { GPS/cell- } \\
\text { phone } \\
\text { tracking }\end{array}$ & $\begin{array}{l}\text { transponder- } \\
\text { based (1) }\end{array}$ & $\begin{array}{l}\text { Airborne } \\
\text { sensors }\end{array}$ & AVL \\
\hline \multirow{9}{*}{$\begin{array}{l}\text { Data collection } \\
\text { capabilities }\end{array}$} & (Point) flows & $\sqrt{ }$ & $\sqrt{ }$ & $\sqrt{ }$ & & & $\sqrt{ }{ }^{c}$ & & \\
\hline & (Point) speeds & $\sqrt{ }$ & $\sqrt{ }$ & $\sqrt{ }$ & & (3) & & & \\
\hline & Occupancies & $\sqrt{ }$ & & $\sqrt{ }$ & & & & & \\
\hline & Subpath flows & & & & $\sqrt{ } \mathrm{a}^{2}$ & $\sqrt{ }$ & $\sqrt{ } \mathrm{a}, \mathrm{c}$ & $\sqrt{ }$ & $\sqrt{b}$ \\
\hline & $\begin{array}{l}\text { Route choice } \\
\text { fractions }\end{array}$ & & & & $\sqrt{ } \mathrm{a}^{2}$ & $\sqrt{ }$ & $\sqrt{ }{ }^{\mathrm{a}, \mathrm{c}}$ & $\sqrt{ }$ & \\
\hline & OD flows & & & & $\sqrt{ }{ }^{\mathrm{a}}$ & $\sqrt{ }$ & $\sqrt{ } \mathrm{a}^{\mathrm{c}} \mathrm{c}$ & $\sqrt{ }$ & \\
\hline & Travel times & & & & $\sqrt{ } a^{a}$ & $\sqrt{ }$ & $\sqrt{ }{ }^{\mathrm{a}, \mathrm{c}}$ & $\sqrt{ }$ & $\sqrt{b}$ \\
\hline & $\begin{array}{l}\text { Vehicle } \\
\text { classification }\end{array}$ & $\sqrt{ }$ & $\sqrt{ }$ & $\sqrt{ }$ & & & $\sqrt{ }$ & $\sqrt{ }$ & \\
\hline & Paths & & & & (2) & $\sqrt{ }$ & (2) & $\sqrt{ }$ & $\sqrt{ }{ }^{a}$ \\
\hline
\end{tabular}

(1) This technology could be used to collect practically any type of information from the vehicle (including speed profiles, origin, destination, and path). In this table, only the information that can be collected by "dumb" transponders, that simply report a unique vehicle signature, is reported

(2) Possible with a dense network of detectors

(3) When combined with GPS

${ }^{a}$ Data limited by network design

${ }^{\mathrm{b}}$ Data limited by market penetration

${ }^{c}$ indirect; for the sample of equipped vehicles only

locate AVI sensors to minimize the variance of the predicted distribution of network travel time.

Naturally, different data collection technologies have widely varying cost and performance characteristics. While these aspects are very interesting, they cannot all be covered in a single manuscript. The interested reader can refer to the relevant literature, including e.g. Middleton et al. [44, 45] and Leduc [39].

\section{Opportunities for dynamic traffic modeling}

The use of emerging data collection technologies in transport can lead to two broad categories of benefits: (i) facilitating direct applications, such as congestion pricing, automatic incident detection (AID), travel time/ speed measurements and (ii) enhancement of existing modeling capabilities through improved support for model development, calibration, and validation. While the first type of impacts has been well discussed, e.g. the new congestion pricing applications in London, Stockholm and elsewhere, the main focus of this section is on the second type of impact.

Applications exploiting AVI data are nowadays quite common. For example, Ma and Koutsopoulos [41] use data collected from a plate matching system (i.e. point-to- point data) in Stockholm, Sweden for the on-line estimation and prediction of travel times in urban areas. The system is based on video cameras placed at various intersections and software with image processing capabilities to match the plate numbers of vehicles as they cross equipped intersections. The data are collected in an urban area, and hence exhibit very high noise. The proposed approach emphasizes the need to properly identify outliers (for example, cars temporarily parked on an urban street). Similarly, Park et al. [54] use real-time AVI data for the prediction of link travel times for one to five (5-min) time periods ahead. The authors employed a spectra basis artificial neural network that utilizes a sinusoidal transformation technique to increase the linear separability of the input features.

While the use of single data sources alone provides interesting applications, the biggest opportunity is the fusing of all available resources under the Dynamic Traffic Assignment (DTA) framework for off-line and on-line calibration of DTA models [19, 20, 42]. The integration of diverse sources of data, which can improve the performance of the various algorithms, is a great opportunity. For example, the calibration of new, emerging model systems, can benefit from data fusion from diverse sources, as each measurement may be more suitable to the calibration of some subset of parameters. 
Point-to-point travel times are valuable for assessing and validating travel times (which is an important expected output of the simulation model), but do not directly provide information on traffic volumes. On the other hand, point flow measurements may not provide particularly rich point-to-point information, but may provide accurate flow data.

In recent years a number of advanced model systems, using simulation-based dynamic traffic assignment (DTA) concepts, have emerged to support network state estimation and prediction [19, 20, 42]. This capability, in turn, enables a number of traffic management applications, such as traffic information generation and dynamic congestion pricing.

DTA systems are typically designed to reside in TMCs and support real-time applications such as the on-line evaluation and implementation of guidance and control strategies, incident management and control, and the support of emergency response operations. DTA models can also be used for short-term planning applications. A DTA system integrates historical data and information from multiple sensor sources to perform two main functions [19, 20]:

- State estimation, during which real-time surveillance information is combined with historical data and a priori parameter values to capture current traffic conditions, using detailed traffic information from instrumented portions of the network to infer the conditions in the parts for which no real-time information is available, and

- Prediction-based information generation, aimed at generating unbiased and consistent traffic information for dissemination to travelers.

In order to provide the above functionality, DTA systems are a synthesis of a number of models and algorithms. DTA systems use detailed travel demand and network supply simulators to synthesize multiple sources of information and perform state estimation and prediction. The demand simulator simulates network-wide demand patterns through time-dependent origin-destination (OD) matrices, and captures the travel choices of individual motorists (e.g. route choice). The supply simulator is usually based on mesoscopic or macroscopic models that represent traffic dynamics using speeddensity relationships and queuing theory. (e.g. [19, 20, 42]). Speed-density relationships depend on location-specific parameters, such as type of facility, number of lanes, lane width, slope, surroundings, as well as weather and time of day factors, reflecting different driving behaviors (e.g. experienced drivers during commute periods). In a DTA model, the complex demand-supply interactions are represented by detailed algorithms that estimate current network state, predict future conditions, and generate anticipatory route guidance and control strategies.
DTA systems combine individual models into a complex system with many inputs and parameters. The proper calibration of these models and inputs is essential to improving their ability to accurately predict future conditions and generate consistent route guidance. Calibration can take place off-line and on-line, with the two modes being synergetic and interacting, as shown in Fig. 1 (explained in the subsections 3.1 and 3.2). An integrated framework for calibration of dynamic traffic models is presented in more detail in Antoniou et al. [5].

AVI data have several applications in traffic management and many more are certain to emerge as these data become more widely available, reliable, and accessible. Representative examples in this field are presented in the remainder of this section.

\subsection{Off-line calibration of dynamic traffic models}

Off-line calibration involves the estimation of variables such as time-varying OD flows, route choice model parameters, other parameters used by the OD estimation and prediction modules and, depending on the nature of the supply simulator, segment capacities and speed-density functions. The variables must be determined such that the model's outputs match the ground reality reflected in archived measurements such as time-varying traffic counts observed across several days. The estimated model inputs and parameters represent average or expected traffic conditions, encapsulating the conditions encountered in the archived data over several prior days. For this reason, they may be viewed as a database of historical estimates.

Stratification of the historical database could allow the off-line calibration of multiple sets of parameters, each corresponding to different prevailing conditions observed in the data. For example, a different set of model inputs and parameters may be created for various combinations of weather conditions, days of the week, seasons and special

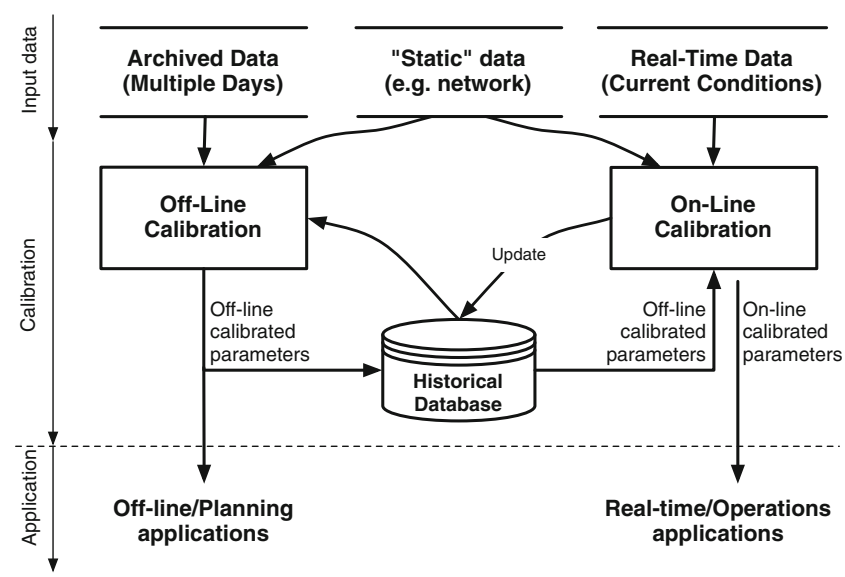

Fig. 1 The role of off-line and on-line calibration 
events. It is assumed that the archived traffic data contain at least a few days of measurements for each combination of factors that is deemed necessary for the modeling exercise. Given a particular combination of factors, the parameters from the closest matching category in the historical database can be quickly selected to accurately assess expected traffic patterns. The historical database has the added advantage of conveniently and efficiently incorporating several days of prior data into the model. If the conditions on a given day are found to deviate from these historical estimates, on-line adjustments may be executed, as explained in the following section.

Complex traffic simulation and assignment models have been developed for various transportation planning and traffic management applications. The state of the art of such models includes simulation-based approaches such as MITSIMLab [61, 62], TransModeler [63], DynaMIT [19, 20], DYNASMART [42], RENAISSANCE [59], DynusT [27], Dynameq [43] and VISTA [25]. The importance and complexity of model calibration has been recognized and documented. However, existing approaches often rely on simplifications and heuristics that limit the accuracy and efficiency of calibration (e.g. sub-problems are solved sequentially, the complex relationships between model parameters and data are approximated, or the use of approximations restricts the data that can be used.

Recent research [10-14] has developed calibration methods that simultaneously estimate all model inputs and parameters, while using the outputs of the model directly (instead of approximating the linkage between the calibration variables and the data). Methodologies to incorporate and efficiently exploit newer data sources are already being developed. For example, state-of-the-art traffic simulation models have been calibrated using speed data in addition to loop detector counts $[10,13]$. The resulting estimates are shown to be more accurate in replicating the observed data due to the use of additional speed information. The results for a case study in Los Angeles, California using the DynaMIT DTA model showed improvements of $45 \%$ in fit to freeway speeds and $37 \%$ in fits to arterial speeds when both count and speed data were used for the calibration (over the base case where only counts were used). It is noted that the improvement in the fit to speed is of particular interest in applications of route guidance and ATIS, since a greater accuracy in speed estimation is expected to translate to better estimates of travel times.

The discussion above has focused on improving the quality of off-line calibration for a given day of traffic measurements. Since traffic conditions can vary significantly across days, the off-line calibration process can be used to create a historical database. For example, parameter sets may be calibrated by day of the week, weather conditions and season, or for specific special events such as concerts, trade shows or sporting events. The process of creating a historical database can benefit significantly from the availability of more advanced sources of traffic measurements. For example, point-to-point speeds or travel times from AVL technology are better reflections of congestion along a link than measurements at a single location. Airborne sensors can also provide data on queue lengths and traffic density, which can improve a dynamic model's ability to estimate and predict congestion. Similarly, route choice model parameters may be fine-tuned using subpath flow data that contains valuable information about drivers' choices along an entire stretch of roadway. Classification counts can be used to calibrate OD tables by vehicle class (e.g. cars, trucks, buses) and vehicle type (e.g. single occupancy, high occupancy), while weather data collected simultaneously with traffic data can aid in stratifying the historical database accordingly.

\subsection{On-line calibration of dynamic traffic models}

Originally, the scope of on-line calibration was limited to OD estimation [1], using link counts as measurements, as that problem could be formulated as a linear problem and thus solved efficiently [6, 8]. More recently, Antoniou et al. [4] have presented a methodology that extends this scope to all model parameters and allows any available type of surveillance information to be used as measurement.

On-line calibration exploits the continuous flow of surveillance information to allow the real-time, dynamic adjustment of model inputs and parameters for each time interval. Using the off-line calibration as a starting point, on-line calibration fine-tunes the model parameters so that they capture the prevailing traffic conditions more accurately, and can therefore lead to better predictions.

The new data sources provide unique opportunities to enhance models for decision support in Dynamic Traffic Management, such as the emerging models presented in the previous section. Richer information can enhance this framework in multiple ways. First, the off-line calibration algorithms will have richer data on which to build the historical database. The supply simulator may use datadriven traffic dynamics models to replicate the movement of vehicles in the network, since more and richer data will be available. The on-line calibration will also benefit from the richer and more reliable surveillance data available over the short horizon that it uses for refinement of a priori parameter values.

A number of studies use AVI data in the context of OD estimation and prediction. Ashok [7] introduced the notion of direct measurements for the incorporation of probe vehicle information for the solution of the OD estimation and prediction problem. Van der Zijpp [67, 68] combined 
volume counts with trajectory information obtained from automated license-plate surveys for the estimation of OD flows. Dixon and Rilett [29] propose a method for using sample link choice proportions and sample OD matrix information derived from AVI data sampled from a portion of vehicles to estimate population OD matrices with the AVI data collection points acting as the origins and destinations. Dixon and Rilett [30] extend this approach to estimate ramp-to-ramp freeway OD volumes, and demonstrate the approach using a large urban freeway network equipped with an AVI system. Kwon and Varaiya [37] develop a statistical OD estimation model using partially observed vehicle trajectories obtained with vehicle reidentification or automatic vehicle identification (AVI) techniques such as electronic tags. Antoniou et al. [2] present a methodology for the incorporation of AVI information into the OD estimation and prediction framework, which is generalized by Antoniou et al. [3] into a flexible formulation that can incorporate a large range of additional surveillance information. Zhou and Mahmassani [66] propose a dynamic origin-destination (OD) estimation method to extract valuable point-to-point split-fraction information from automatic vehicle identification (AVI) counts without estimating market-penetration rates and identification rates of AVI tags.

The additional data are useful not only on their own merit, but also as used in the above applications to improve the accuracy of the estimation and prediction of OD flows. For example, Antoniou et al. [3] report improvements in terms of normalized root mean square error (RMSN) of more than $40 \%$ for OD estimation and up to three-step prediction (i.e. looking up to three time intervals ahead), and $37 \%$ for four-step prediction, when AVI data are added to count data (Table 2). Besides only relying on link point counts for the OD estimation, in this case study it is assumed that AVI detectors are located at three fixed locations on the network. Subsequent detections of the transponders of the equipped vehicles by these detectors provide two subpath flow measurements. This information is added to the formulation of the OD estimation and prediction problem resulting in the improvements shown in Table 2. For more details on the assumptions, the network geometry and the formulation, the reader is referred to Antoniou et al. [3].

\section{Opportunities for advanced/new applications}

The sensors described in the previous sections collect traffic data that both improve models and enhance existing applications, for example improved traffic prediction can be used in many applications such as fleet monitoring and richer travel planners. However it is important to also point out that general sensor developments also provide the opportunity to collect and use other types of data related to infrastructure and the environment that could enhance traffic management to support broader and multiple objectives such as operation taking into consideration impact to the environment and the infrastructure.

\subsection{Examples of condition monitoring sensors}

There is potential for significant benefits through the integration of traffic data with other types of data. For example, the sensor network can be enlarged to encompass information about the condition of infrastructure that affects traffic operations, such as roads, bridges and tunnels. This type of technologies may include:

- Environmental monitoring: Environmental sensors and weather stations could be used for the timely determination of adverse weather conditions (such as heavy storms, extreme temperatures or low visibility due to fog), icy or wet road surface or high emissions concentrations. This information can be used to prevent accidents, and can also be incorporated into traffic management efforts. Sensors in this category include:

- Weather stations: Weather information is commonly provided by TMC websites and other sources. The strategic placement of weather stations near the roadway network provides accurate monitoring of prevailing conditions. Besides standard weather information (temperature, barometric pressure, rainfall, wind direction and intensity), dedicated traffic weather stations may provide additional information, such as pavement condition (icy/wet) and visibility (e.g. due to fog) [22].

- Air quality stations: Maps of traffic-related air pollution can be combined in real-time with traffic information to guide traffic management with
Table 2 OD flow estimation and prediction improvement due to AVI data

\begin{tabular}{lccccc}
\hline & Estimation & \multicolumn{2}{l}{ Prediction (RMSN) } & \\
\cline { 3 - 5 } & & One-step & Two-step & Three-step & Four-step \\
\hline Counts & 0.250 & 0.254 & 0.261 & 0.268 & 0.279 \\
Counts + AVI & 0.133 & 0.139 & 0.146 & 0.159 & 0.176 \\
$\%$ Improvement & 47 & 45 & 44 & 41 & 37 \\
\hline
\end{tabular}


multiple objectives, such as minimization of travel time and emissions. Briggs et al. [23] and Gulliver and Briggs [34] present examples of the combination of air pollution data with traffic data.

- Early flood warning systems: Flooding in rivers and lakes can adversely impact nearby roadways. Early warning systems could provide traffic management operators with sufficient time to divert traffic in such events [58].

- Infrastructure condition monitoring: Embedded sensors and scanning techniques could monitor and diagnose the condition of pavement, bridges, tunnels, highway overpasses, retaining walls, etc. These data could be used to predict and avert potential failures. Early warnings could be used to schedule preventative maintenance activities. Furthermore, early warning of failures can be used to create diversion strategies that direct traffic away from affected infrastructure, before traffic conditions become adverse. This may also allow emergency personnel to reach the affected area without problems. Sensors in this category include:

- Strain meters and gauges, (piezoelectric, capacitive, etc.) accelerometers: provide seismic and structural monitoring of structures close to roadways [40].

- Fiber-optic sensors: versatile, flexible, and inexpensive sensors that can accurately monitor structural condition [24].

\subsection{Applications of integrated traffic and condition monitoring sensors}

The fusion of condition information with traffic data can result in better and more responsive dynamic traffic management applications with a richer data background. Barnett and Benekohal [17] investigate the accident reduction benefits of combining weigh-in-motion and AVI technologies in order to allow equipped trucks to by-pass weigh stations. The authors developed a model that can predict potential accident reductions around weight stations at varying levels of WIM and AVI usage. Benekohal et al. [21] present the truck travel time savings that can be obtained from a preclearance system that combines AVI and WIM technologies.

Advanced measuring technologies can greatly benefit the management of transportation networks during emergency evacuations. Such situations usually consist of various components discussed earlier in this section. For example, condition-monitoring sensors can provide information about the operational status of key transportation infrastructure such as bridges and tunnels, so that first responders and managers can route traffic onto safe routes. Environmental sensors may provide feedback about the propagation of chemical spills and plumes. Similarly, early flood warning systems could provide traffic management operators with sufficient time to divert traffic in such events [58].

People are generally expected to react differently when faced with uncertain and potentially life-threatening situations. In the context of transportation network management, this may translate into choices of evacuation mode, routes and destinations. Technology can play a key role in measuring the collective effect of these individual decisions in real-time. Traffic detectors can fill in the crucial links about the development of bottlenecks and the spatial and temporal availability of spare capacity so that the evacuation demand can be handled in the most efficient manner.

As data collection technologies become more commonplace and accessible, further applications are expected to emerge. For example, recent research investigates the applicability of AVI technologies for measuring border delay and crossing times at the U.S./Mexico border [69] and for congestion pricing at border crossings [9]. Distance-based user charging [65] and pay-as-you-go insurance are other applications that rely on the proliferation of innovative data collection methods.

\section{Conclusion}

The objective of this paper is to provide an overview of emerging data collection technologies and their impact on traffic management applications. Several existing and emerging surveillance technologies are being used for traffic data collection. Each of these technologies has different technical characteristics and operating principles, which determine the types of data collected, accuracy of the measurements, levels of maturity, feasibility and cost, and network coverage. This paper reviews the different sources of traffic surveillance data currently employed, and the types of traffic management applications they may support. Traffic sensor technologies are classified based on their functionality into three broad categories as point, point-to-point and area-wide.

Clearly, the applications of advanced information for traffic management applications are numerous, and more than can be included in a single paper. While considerable advances have taken place in methodological aspects of transportation management applications, data surveillance and collection was until recently revolving around conventional loop detectors. From the review presented in this paper, it becomes apparent that numerous emerging technologies are becoming increasingly available. These technologies provide additional types of data that were previously impossible, or too difficult, to collect, with practical advantages over conventional data collection mechanisms (e.g. lower cost, higher reliability and accuracy).

Opportunities offered by emerging traffic data collection technologies in the context of dynamic traffic data 
modeling are presented, focusing on their role in the development and application of more effective (off-line and on-line) calibration methodologies in simulation-based traffic management systems. Advanced applications, such as those that could be obtained from the integration of traffic and condition monitoring sensors are also discussed.

Open Access This article is distributed under the terms of the Creative Commons Attribution License which permits any use, distribution and reproduction in any medium, provided the original author(s) and source are credited.

\section{References}

1. Antoniou C, Ben-Akiva M, Bierlaire M, Mishalani R (1997) Demand simulation for dynamic traffic assignment. Proceedings of the 8th IFAC Symposium on Transportation Systems, Chania, Greece, June

2. Antoniou C, Ben-Akiva M, Koutsopoulos HN (2004) Incorporating automated vehicle identification data into origin-destination estimation. Transportation Research Record, No. 1882, pp 37-44, Washington D.C.

3. Antoniou C, Ben-Akiva M, Koutsopoulos HN (2006) Dynamic traffic demand prediction using conventional and emerging data sources. IEE Proc Intell Transp Syst 153(1):97-104

4. Antoniou C, Ben-Akiva M, Koutsopoulos HN (2007) Non-linear Kalman filtering algorithms for on-line calibration of dynamic traffic assignment models. IEEE Trans Intell Transp Syst 8 (4):661-670

5. Antoniou C, Balakrishna R, Koutsopoulos HN, Ben-Akiva M (2010) Calibration methods for simulation-based dynamic traffic assignment systems. Accepted for publication in International Journal of Modeling and Simulation

6. Ashok K, Ben-Akiva M (1993) Dynamic O-D matrix estimation and prediction for real-time traffic management systems. In: Daganzo C (ed) Transportation and traffic theory. Elsevier Science Publishing, Oxford, pp 465-484

7. Ashok K (1996) Estimation and prediction of time-dependent origin-destination flows, $\mathrm{PhD}$ thesis, Massachusetts Institute of Technology

8. Ashok K, Ben-Akiva M (2000) Alternative approaches for realtime estimation and prediction of time-dependent origindestination flows. Transp Sci 34(1):21-36

9. Baker RT, Ungemah D, Boyd MA (2008) Borders, barriers and benefits. The realities of congestion pricing at El-Paso commercial border crossings. Proceedings of the 87th Annual Meeting of the Transportation Research Board Washington, D.C

10. Balakrishna R, Ben-Akiva M, Koutsopoulos HN (2006) Timedependent origin-destination flows without assignment matrices, Second International Symposium of Transport Simulation (ISTS06), 4-6 September

11. Balakrishna R (2006) Off-line calibration of dynamic traffic assignment models, $\mathrm{PhD}$ thesis, Massachusetts Institute of Technology

12. Balakrishna R, Ben-Akiva M, Koutsopoulos HN (2007) Simultaneous off-line demand-supply calibration for simulation-based dynamic traffic assignment models. 11th World Conference on Transport Research (WCTR), June 24-28, Berkeley

13. Balakrishna R, Antoniou C, Ben-Akiva M, Koutsopoulos HN, Wen Y (2007) Calibration of microscopic traffic simulation models: methods and application. Transp Res Rec 1999:198-207
14. Balakrishna R, Ben-Akiva M, Koutsopoulos HN (2007) Off-line calibration of dynamic traffic assignment: simultaneous demandsupply estimation. Transp Res Rec 2003:50-58

15. Bar-Gera H (2007) Evaluation of a cellular phone-based system for measurements of traffic speeds and travel times: a case study from Israel. Transp Res Part C: Emerg Technol 15(6):380-391

16. Barcelo J, Montero L, Marques L, Carmona C (2010) Travel time forecasting and dynamic OD estimation in freeways based on Bluetooth traffic monitoring. Proceedings of the 89th Annual Meeting of the Transportation Research Board, Washington, D.C

17. Barnett JC, Benekohal RF (1999) Accident reduction effects of using weigh-in-motion and automatic vehicle identification for mainline bypass around truck weigh stations. Transp Res Rec 1655:233-240

18. Bekhor S, Hirsh M, Nimre S, Feldman I (2008) Identifying spatial and temporal congestion characteristics using mobile phone data. Proceedings of the 87th Annual Meeting of the Transportation Research Board, Washington, D.C

19. Ben-Akiva M, Bierlaire M, Koutsopoulos HN, Mishalani R (2002) Real-time simulation of traffic demand-supply interactions within DynaMIT. In Gendreau M, Marcotte P (Eds.), Transportation and network analysis: current trends, pages 19-36. Kluwer Academic Publishers. Miscellenea in honor of Michael Florian

20. Ben-Akiva M, Koutsopoulos HN, Antoniou C, Balakrishna R (2010) Traffic simulation with DynaMIT. In: Barcelo J (ed) Fundamentals of traffic simulation. Springer, pp. 363-398

21. Benekohal RF, El-Zohairy YM, Wang S (2000) Truck travel time around weigh stations. Effects of weigh in motion and automatic vehicle identification systems. Transp Res Rec 1716:135-143

22. Boselly SE (2001) Benefit/cost study of Road Weather Information Systems (RWIS) and anti-icing technologies. Final Report, prepared for the National Cooperative Highway Research Program, Transportation Research Board

23. Briggs DJ, de Hoogh C, Gulliver J, Wills J, Elliot P, Kingham S, Smallbone K (2000) A regression-based method for mapping traffic-related air pollution: application and testing in four contrasting urban environments. Sci Total Environ 253(13):151-167, 15 May 2000

24. Calvert SG, Conte JP, Moaveni B, Schulz WL, de Callalfon R (2003) Real time damage assessment using fiber optic grating sensors. Proc SPIE 5278:110-121

25. Chang E, Ziliaskopoulos A (2007) A simulation-based dynamic intermodal network equilibrium algorithm. In: Network Science, Nonlinear Science and Infrastructure Systems, International Series in Operations Research \& Management Science, 2007, Volume 102, pp. 201-222

26. Chen A, Chootinan P, Pravinvongvuth S (2004) Multiobjective model for locating automatic vehicle identification readers. Transp Res Rec 1886:49-58

27. Chiu Y-C, Zhou L, Song H (2009) Development and calibration of anisotropic mesoscopic simulation model for uninterrupted flow facilities. Transp Res Part B: Methodol 44(1):152-174

28. Coifman B, Krishnamurthy S (2007) Vehicle reidentification and travel time measurement across freeway junctions using the existing detector infrastructure. Transp Res Part C 15:135-153

29. Dixon MP, Rilett LR (2000) Real-time origin-destination estimation using automatic vehicle identification data'. Proceedings of the 79th Transportation Research Board Meeting, Washington, D.C

30. Dixon MP, Rilett LR (2005) Population origin-destination estimation using automatic vehicle identification and volume data. J Transp Eng 131(2):75-82

31. Federal Highway Administration (FHWA) (1998) Travel time data collection handbook. Chapter 4. License Plate Matching Techniques. Report FHWA-PL-98-035

32. Furth P, Hemily B, Muller T, Strathman J (2003) Uses of archived AVL-APC data to improve transit performance and management: review and potential, TCRP Web Document 23 
33. Gomez A, Shen LD (1998) Benefits of transit AVL and transit AVL implementation in the U.S. Proceedings of the 78th Annual Meeting of the Transportation Research Board, Washington, D.C

34. Gulliver J, Briggs DJ (2005) Time-space modeling of journeytime exposure to traffic-related air pollution using GIS. Environ Res 97(1):10-25

35. Haghani A, Hamedi M, Sadabadi KF, Young S, Tarnoff P (2010) Freeway travel time ground truth data collection using bluetooth sensors. Proceedings of the 89th Annual Meeting of the Transportation Research Board, Washington, D.C

36. Hellinga B, Fu L (1999) Assessing expected accuracy of probe vehicle travel time reports. J Transp Eng 125(6):524-530

37. Kwon J, Varaiya P (2005) Real-time estimation of O-D matrices with partial trajectories from electronic toll collection tag data. Transp Res Rec 1923:119-126

38. Laborczi P, Nowotny B, Linauer M, Schneider M, Karim R, Leihs, D (2004) Optimal route guidance based on floating car data. Proceedings of the 10th World Conference on Transport Research (WCTR), Istanbul, July

39. Leduc G (2008) Road traffic data: collection methods and applications. working papers on energy, transport and climate change, JRC technical notes, European Commission

40. Lynch JP, Sundararajan A, Law KH, Sohn H, Farrar CR (2003) New opportunities for structural monitoring: wireless active sensing. Proceedings of the International Workshop on Advanced Sensors, Structural Health Monitoring, and Smart Structures. Keio University, Tokyo, Japan, November 10-12

41. Ma X, Koutsopoulos HN (2008) A new online travel time estimation approach using distorted automatic vehicle identification data, Proceedings of the 11th International IEEE Conference on Intelligent Transportation Systems (CD ROM), Beijing, China

42. Mahmassani HS (2001) Dynamic network traffic assignment and simulation methodology for advanced system management applications. Netw Spacial Econ 1(3):267-292

43. Mahut M, Florian M (2010) Traffic simulation with dynameq. In J Barcelo (ed) Fundamentals of traffic simulation, Springer, pp. 323-361

44. Middleton D, Jasek D, Parker R (2000) Evaluation of some existing technologies for vehicle detection, Project Summary Report 1715-S, Texas Transportation Institute

45. Middleton D, Gopalakrishna D, Raman M (2003) Advances in traffic data collection and management. Intelligent Transportation Systems Report No. 13766, US Department of Transportation

46. Minami M, Kato R, Hagiwara T, Araki K, Nagata Y, Takitani K (2008). Development of a visibility estimation model based on visibility information from road images captured in winter. Proceedings of the 87th Annual Meeting of the Transportation Research Board, Washington, D.C

47. Mirchandani PB, He Y (2008) Sensor locations on a network to predict travel times. Proceedings of the 87th Annual Meeting of the Transportation Research Board, Washington, D.C

48. Ndoye M, Totten V, Carter B, Bullock DM, Krogmeier JV (2008) Vehicle detector signatures processing and vehicle reidentification for travel time estimation. Proceedings of the 87th Annual Meeting of the Transportation Research Board, Washington, D.C

49. Nooralahiyan A, Kirby H, McKeown D (1998) Vehicle classification by acoustic signature. Math Comput Model 27(9-11):205-214

50. Oh S, Ritchie SG, Oh C (2002) Real time traffic measurement from single loop inductive signatures. Proceedings of the 81 st Annual Meeting of the Transportation Research Board, Washington, D.C
51. Oh C, Ritchie SG, Jeng S-T (2004) Vehicle reidentification using heterogeneous detection systems. Proceedings of the 3rd Annual Meeting of the Transportation Research Board, Washington, D.C

52. Okunieff, Paula E (1997) Synthesis of Transit Practice 24: AVL Systems for Bus Transit, Transportation Cooperative Research Program, TRB, National Research Council

53. Pack ML, Smith, BL, Scherer WT (2003) An automated camera repositioning technique for integrating video image vehicle detection systems with freeway CCTV systems. Proceedings of the 82th Annual Meeting of the Transportation Research Board, Washington D.C

54. Park D, Rilett LR, Han G (1999) Spectral basis neural networks for real-time travel time forecasting. J Transp Eng 125(6):515-523

55. Quayle S, Koonce P, DePencier D, Bullock D (2010) Arterial performance measures using MAC readers: Portland pilot study. Proceedings of the 89th Annual Meeting of the Transportation Research Board, Washington, D.C

56. Quiroga C, Perez M, Venglar S (2002) Tool for measuring travel time and delay on arterial corridors. In ASCE 7th International Conference on Applications of Advanced Technology in Transportation, pages 600-607, Cambridge, MA

57. Srinivasan S, Latchman H, Shea J, Wong T, McNair J (2004) Airborne traffic surveillance systems - video surveillance of highway traffic. Proceedings of the ACM VSSN' 04 conference, New York

58. Wang X, Huang S, Du C (1999) Development of a flood warning information system. Proceedings of the 19th Annual ESRI User Conference, San Diego, CA

59. Wang Y, Papageorgiou M, Messmer A (2006) RENAISSANCE A unified macroscopic model-based approach to real-time freeway network traffic surveillance. Transp Res 14 C:190-212

60. Work DB, Bayen AM (2008) Impacts of the mobile internet on transportation cyberphysical systems: traffic monitoring using smartphones. In National Workshop for Research on HighConfidence Transportation Cyber-Physical Systems: Automotive, Aviation and Rail, Washington, DC, November 18-20

61. Yang Q, Koutsopoulos HN (1996) A microscopic traffic simulator for evaluation of dynamic traffic management systems. Transp Res C 4(3):113-129

62. Yang Q, Koutsopoulos HN, Ben-Akiva ME (2000) A simulation model for evaluating dynamic traffic management systems. Transp Res Rec 1710:122-130

63. Yang Q, Morgan D (2006) A hybrid traffic simulation model. Proceedings of the 85th Annual Meeting of the Transportation Research Board, Washington, D.C

64. Yannis G, Antoniou C (2005) Integration of weigh-in-motion technologies in road infrastructure management. J Inst Transp Eng (ITE) 75(1):39-43

65. Zhang L, McMullan BS (2008) Statewide distance-based user charge: the case of Oregon. Proceedings of the 87th Annual Meeting of the Transportation Research Board, Washington, D.C

66. Zhou, Mahmassani (2006) Dynamic origin-destination demand estimation using automatic vehicle identification data. IEEE Trans Intell Transp Syst 7(1):105-114

67. van der Zijpp N (1996) Dynamic origin-destination matrix estimation on motorway networks, $\mathrm{PhD}$ thesis, Delft University of Technology

68. van der Zijpp N (1997) Dynamic origin-destination matrix estimation from traffic counts and automated vehicle identification data. Transp Res Rec 1607:87-94

69. Villa JC, Solari-Terra M (2008) Technology assessment to measure border delay and crossing times at the U.S./Mexico Border. Proceedings of the 87th Annual Meeting of the Transportation Research Board, Washington, D.C 